\title{
Heat transfer enhancement of a single row of tube
}

\author{
Takayuki Tsutsui ${ }^{1, *}$ \\ ${ }^{1}$ Department of Mechanical Engineering, The National Defense Academy, 1-10-20 Hashirimizu, Yokosuka, Kanagawa 238-8686 Japan
}

\begin{abstract}
A rod was positioned upstream of a circular cylinder to enhance its heat transfer and reduce its drag in air stream. The results are increasing the overall heat transfer by $40 \%$ over and reducing the drag by $30 \%$ the value for a single circular cylinder. In the present, this technique applied to a single row of tubes. Heat transfer enhancement and drag reduction are important factor for multi-tube heat exchanger. The present study investigated the heat transfer and surface pressure characteristics of each tube for single row of tubes under various numbers of tubes, pitch and Reynolds number. The tube diameter, D, was $20 \mathrm{~mm}$, and the rod diameter was $5 \mathrm{~mm}$. The distance between the center axes of the rod and the tube was $30 \mathrm{~mm}$. The Reynolds number based on D ranged from $5.3 \times 10^{3}$ to $2.1 \times 10^{4}$. The result is that this technique is effective for heat transfer enhancement and drag reduction of row of tube.
\end{abstract}

\section{Introduction}

Heat transfer enhancement and drag reduction are important in the design of multi-tube heat exchangers. Ribs [for example 1], rib roughness [for example 2] and winglets [for example 3] are well known as good manners. But the machine production on surface of tubes and fin is necessary for them. It has been previously shown that heat transfer and drag can be respectively enhanced and reduced simply by positioning a rod upstream from a circular cylinder. [4, 5] Under the optimum conditions, for a single circular cylinder with a rod positioned upstream, the overall heat transfer is increased by $40 \%$, while the drag of the cylinder is reduced by $30 \%$.

Figure 1 (a) shows the flow visualization of a circular cylinder with a rod under the optimum conditions of $\mathrm{L} / \mathrm{D}=1.5$ and $\mathrm{d} / \mathrm{D}=0.25$, where $\mathrm{D}, \mathrm{d}$, and $\mathrm{L}$ are the cylinder diameter, rod diameter, and distance between the center axes of the rod and the cylinder, respectively. The separated shear layer from the rod reattaches at the front face of the cylinder, and a quasi-static vortex is formed between the rod and the cylinder. For comparison, Figure 1 (b) shows the flow visualization of a circular cylinder without a rod.

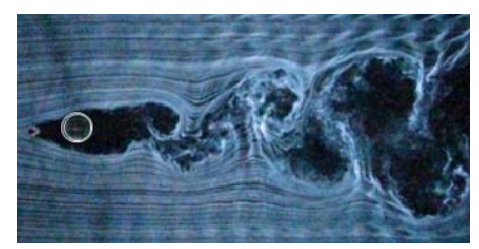

(a)
Figure 2 (a) shows the Nusselt number $(\mathrm{Nu})$ distribution. $\mathrm{Nu}$ is considerably higher at approximately $\phi= \pm 40^{\circ} \sim \pm 50^{\circ}$, which corresponds to the separated shear layer reattachment points. However, $\mathrm{Nu}$ in the frontal stagnation area $\left(\phi= \pm 20^{\circ}\right)$ is lower than for a single cylinder due to the quasi-static vortex. The overall value of $\mathrm{Nu}$ at a Reynold's number of $R e=2.1 \times$ $10^{4}$ is over $40 \%$ higher than the corresponding value for a single cylinder [1]. Figure 2 (b) shows the pressure coefficient (Cp) distribution on the front region $(\phi= \pm 25$ $\left.{ }^{\circ}\right)$. Here $\mathrm{Cp}$ is negative because a quasi-static vortex has formed between the rod and the cylinder. $\mathrm{Cp}$ reaches a maximum at the reattachment region $\left(\phi= \pm 35^{\circ}\right)$ of the shear layer that separated from the rod.

There are many applications of this technique to cylinder clusters in the fields of mechanical, civil and electrical engineering, e.g., in heat exchangers, chimney stacks, and electric transmission lines. One example of such a heat exchanger incorporates several tubes. Heat transfer enhancement and drag reduction are important in multi-tube heat exchangers. Several arrangements of cylinder clusters and multi-tube arrays were reviewed by Zdravkovich [6], and the most effective arrangement for applications of this technique was reported to be a single row of tubes.

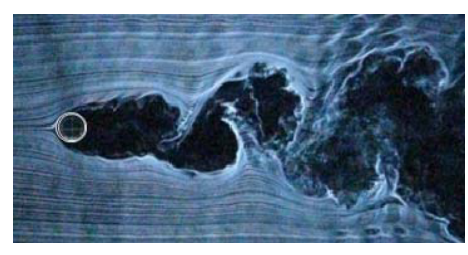

(b)

Fig. 1. Flow visualization of a single cylinder $\left(D=20 \mathrm{~mm}, \mathrm{Re}=1.1 \times 10^{4}\right)(\mathrm{a})$ with and (b) without a rod

tsutsui@nda.ac.jp

(C) The Authors, published by EDP Sciences. This is an open access article distributed under the terms of the Creative Commons Attribution License 4.0 (http://creativecommons.org/licenses/by/4.0/). 
The goal of the present study is to confirm the effectiveness of this technique for multi-tube arrangements. In the present study, we investigate the heat transfer and surface pressure characteristics of each tube in a single row of tubes for various numbers of tubes, pitches, and Reynolds numbers.

\section{Experimental apparatus and methods}

The configuration of the row of tubes and the experimental setup are shown in Figure 3. Experiments were performed in a low-speed wind tunnel with a 400mm-high, 300-mm-wide, and 400-mm-long working section. The tube diameter, D, was $20 \mathrm{~mm}$, and the rod diameter, d, was $5 \mathrm{~mm}$. The longitudinal distance $\mathrm{L}$ between the center axes of the rod and the tube was 30 $\mathrm{mm}$. The ratios $\mathrm{d} / \mathrm{D}$ and $\mathrm{L} / \mathrm{D}$ were 0.25 and 1.5, respectively, which correspond to the optimal conditions for heat transfer enhancement and drag reduction [4, 5]. The pitch, $\mathrm{S}$, between adjacent tubes was varied from 21 to $60 \mathrm{~mm}$. The freestream velocity $U$ was varied between 4 and $16 \mathrm{~m} \mathrm{/} \mathrm{s,} \mathrm{and} \mathrm{the} \mathrm{turbulent} \mathrm{intensity} \mathrm{was}$ approximately $0.4 \%$ in this range. The value of Reynolds number, Re, based on D ranged from $5.3 \times 10^{3}$ to $2.1 \times 10^{4}$.

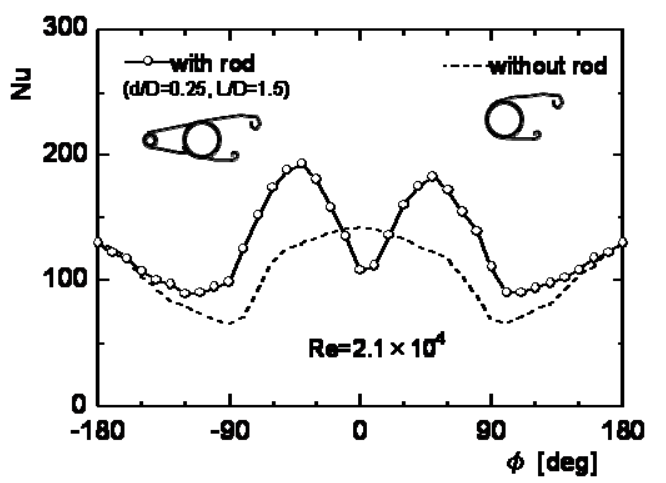

(a)
Figures 4 (a) and 4 (b) show the constant heat flux model and measurement system respectively, used in the present study. The models were used for local heat transfer measurements and were fabricated from 3-mmthick acrylic resin pipe, which was covered with two 8$\mu \mathrm{m}$-thick stainless steel sheets. The sheets were electrically connected in series and were heated by applying a direct current to produce a constant heat flux condition. Copper-constantan (C-C) thermocouples with diameters of $0.1 \mathrm{~mm}$ were attached to the underside of Figures 4 (a) and 4 (b) show the constant heat flux model and measurement system respectively, used in the present study. The models were used for local heat transfer measurements and were fabricated from 3-mmthick acrylic resin pipe, which was covered with two 8$\mu \mathrm{m}$-thick stainless steel sheets. The sheets were electrically connected in series and were heated by applying a direct current to produce a constant heat flux condition. Copper-constantan (C-C) thermocouples with the stainless steel sheets in a spiral arrangement at tendegree intervals. The local temperatures on the surfaces of the tubes were measured simultaneously with respect to the free-stream velocity and gap parameters. The surface pressure on the tubes was measured by a multitube pressure measurement system connected to 0.6-

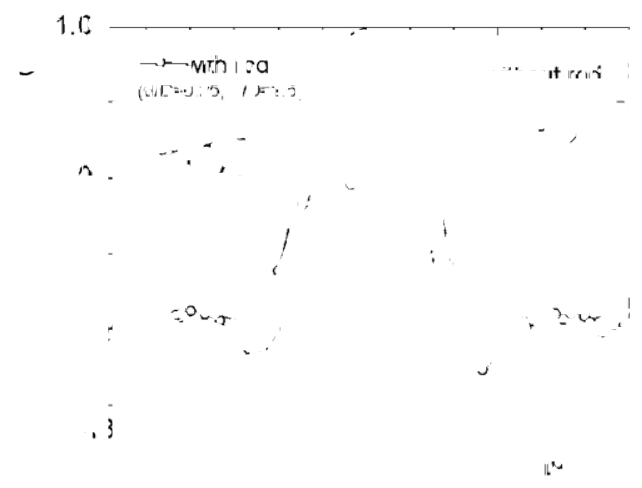

(b)

Fig. 2. Effect of the rod $\left(D=20 \mathrm{~mm}, \mathrm{Re}=2.1 \times 10^{4}\right)$ (a) Nusselt number distribution and
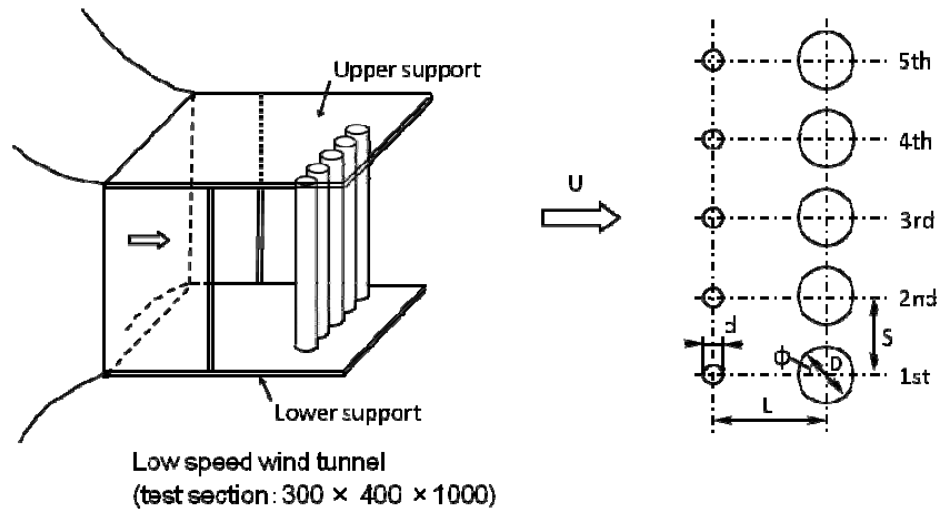

Fig. 3. Coordinate system and experimental setup 


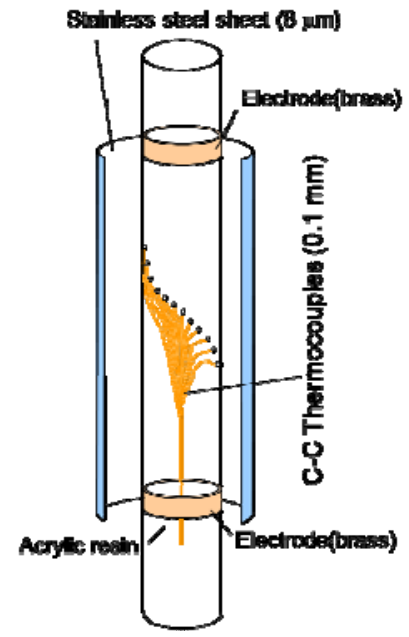

(a)

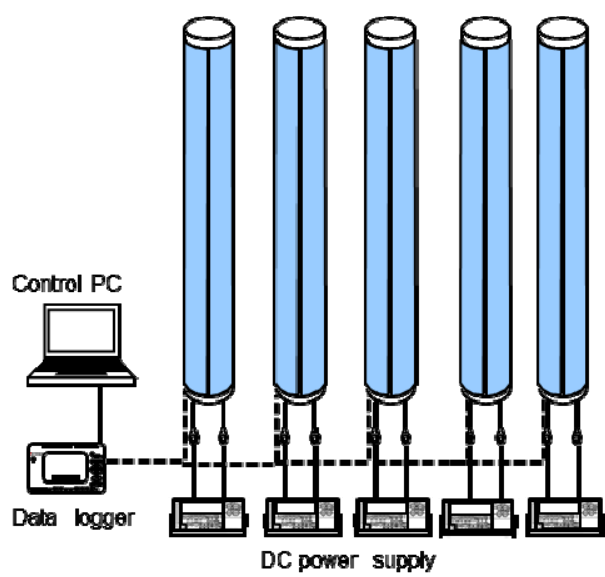

(b)

Fig. 4. Schematic illustrations of (a) Constant heat flux model and (b) measurement system.

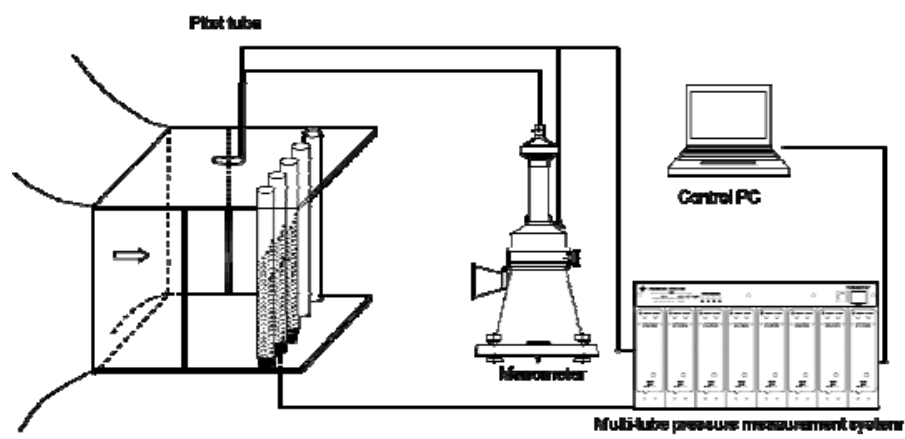

Fig. 5. Schematic illustration of pressure measurement system.

mm-diameter pressure taps in a spiral arrangement at ten-degree intervals on the surfaces of the tubes.

Figure 5 shows the pressure measurement system. We prepared five identical models and measured the surface pressures for all of the tubes simultaneously.

\section{Results and discussion}

\subsection{Flow visualization and wake}

Figures 6 shows photographs of the flow visualizations, velocity, $\mathrm{u} / \mathrm{U}$, and turbulence intensity, $\Delta \mathrm{u} / \mathrm{U}$, profiles in the wakes of rows of two, three, four, and five tubes, where $\mathrm{u}$ and $\Delta \mathrm{u}$ are the velocity and root mean square (RMS) value of fluctuating velocity, respectively. The flow visualizations were obtained using the smoke wire method, and the photographs show the flows with and without rods. The profiles show the velocity and turbulence intensity in the upper and lower parts of the figure. The $\mathrm{u} / \mathrm{U}$ and $\Delta \mathrm{u} / \mathrm{U}$ profiles reveal the differences in the gap flow and the wake for the "with rods' and 'without rods' conditions.

As shown in Figure 6 (a), for the two-tube case without rods, the flow around the tube is a typical biased flow [7]. The gap flow between the tube switches intermittently from one side to the other side of the tubes. For the 'with rods' conditions, the wakes from the rods are biased toward the upper and lower sides (outer sides), and these wakes reattach on the upper and lower sides of the front face of the tube. The gap flow between the tubes becomes biased toward the upper tube. 


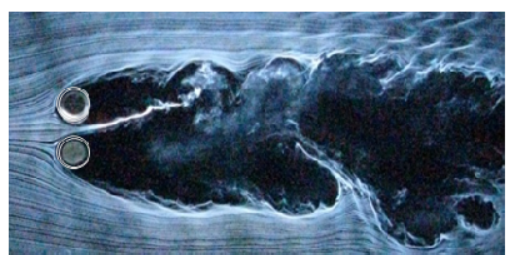

(a) without rods

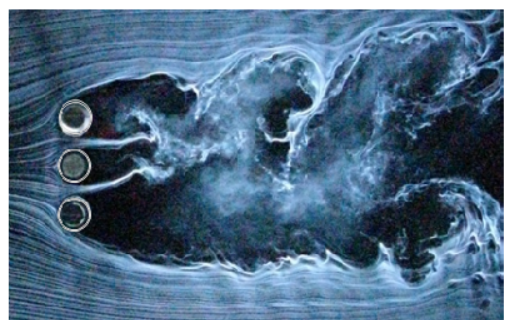

(b) without rods

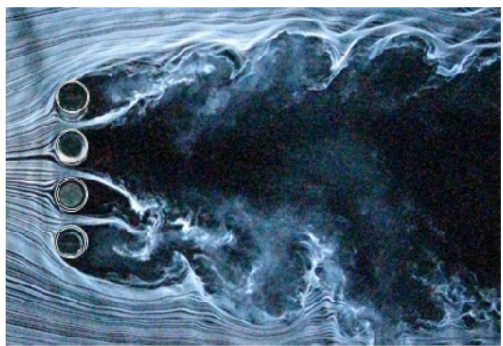

(c) without rods

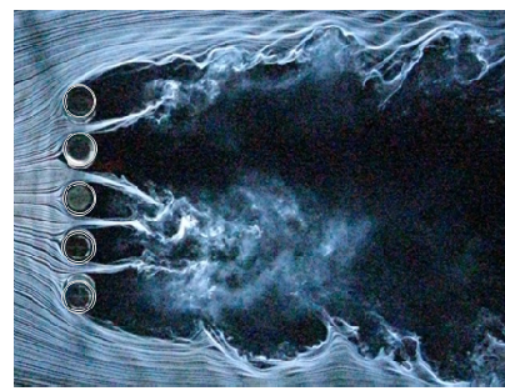

(d) without rods

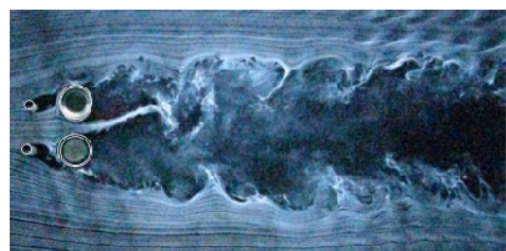

with rods

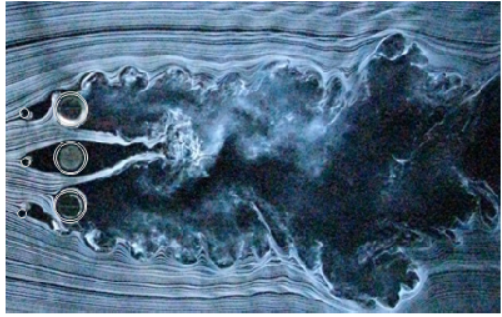

with rods

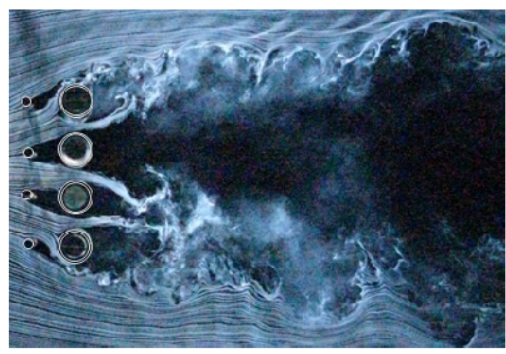

with rods

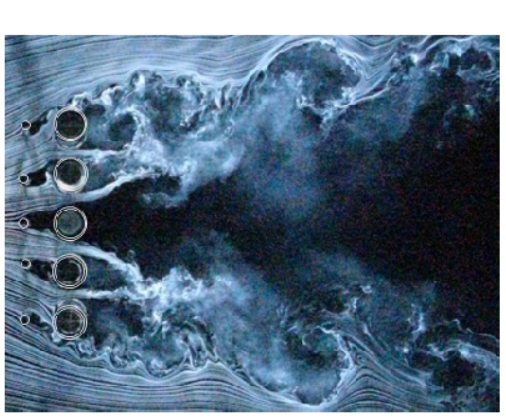

with rods
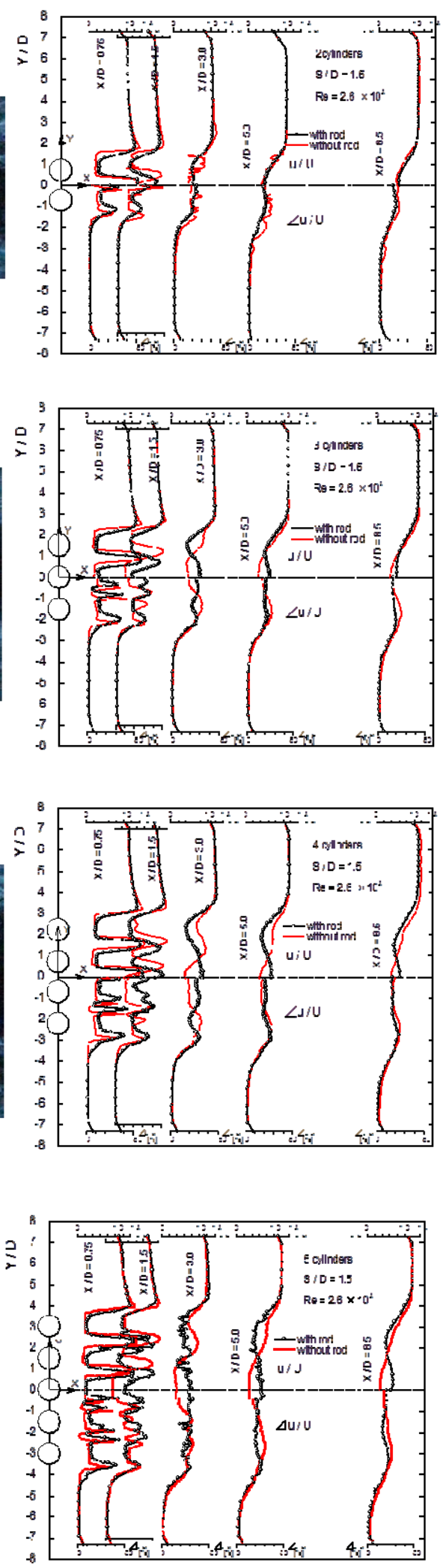

Fig. 6. Flow visualization and wake $\left(\mathrm{D}=20 \mathrm{~mm}, \mathrm{Re}=2.1 \times 10^{4}, \mathrm{~S} / \mathrm{D}=1.5\right)$ for (a) two tubes, (b) three tubes, (c) four tubes, and (d) five tubes. 


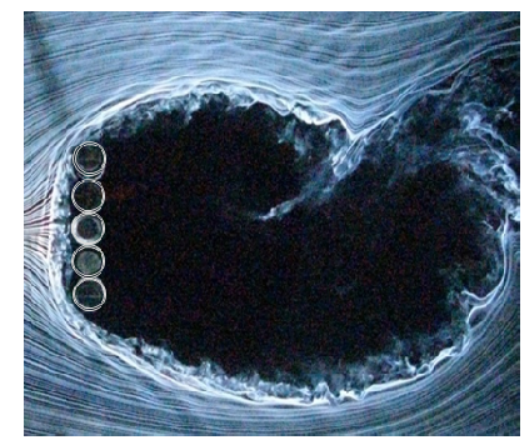

(a) without rods

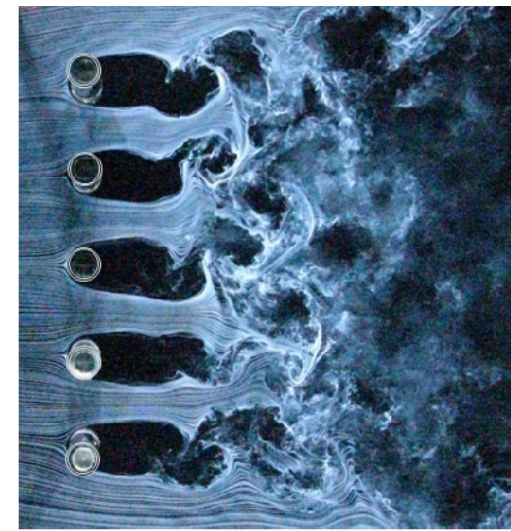

(b) without rods

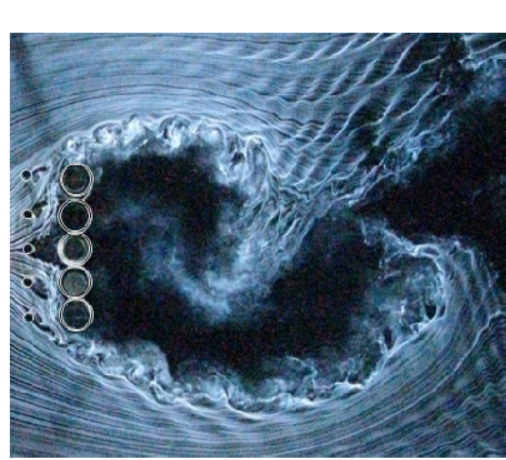

with rods
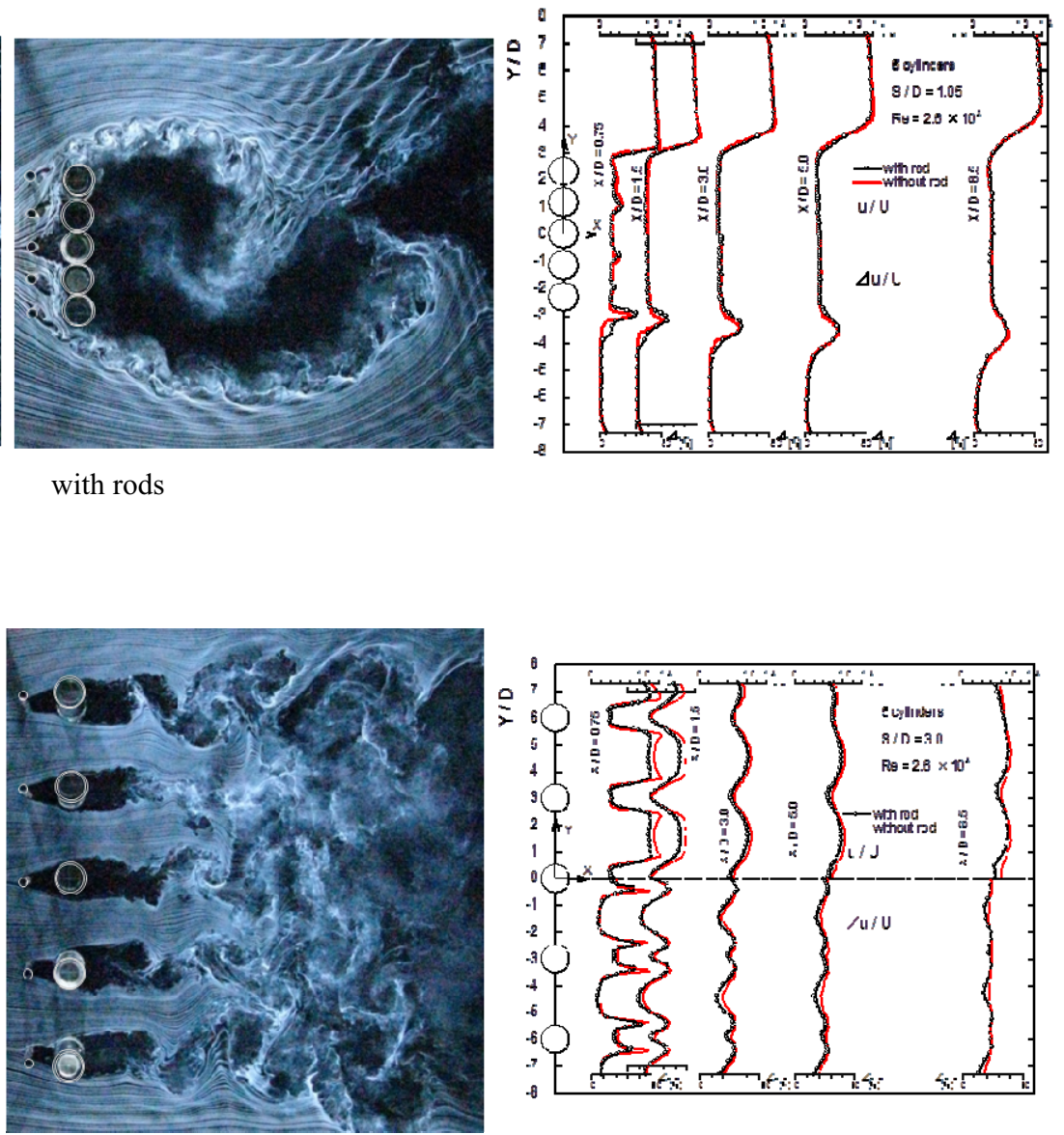

with rods

Fig. 7. Flow visualization and wake (effect of pitch in the five-tube system, $D=20 \mathrm{~mm}, \mathrm{Re}=2.1 \times 10^{4}$ ) for (a) $\mathrm{S} / \mathrm{D}=1.05$ and (b) $\mathrm{S} / \mathrm{D}=3.0$.

For rows of three- to five-tube without rods, as biased in every case. For the 'with rods' conditions, the separated shear layer from the center rod or from a more centrally located rod reattaches on the front face of the tube positioned directly behind the rod. The wakes of the uppermost and lowermost rods are biased toward the upper and lower sides, respectively.

Based on the flow visualizations and the $\mathrm{u} / \mathrm{U}$ and $\Delta \mathrm{u} / \mathrm{U}$ profiles, as a general tendency, for the 'with rods' conditions, the gap flow is biased toward the center, and the wake is narrower than that for the 'without rods' conditions

Figure 7 shows the flows for two different pitch conditions for the rows of rods and tubes for the case of five tubes. For $\mathrm{S} / \mathrm{D}=1.05$, a single vortex street is formed behind the tubes. There is no gap flow between the tubes and the differences in the profiles of $\mathrm{u} / \mathrm{U}$ and $\Delta \mathrm{u} / \mathrm{U}$ between the cases with and without rods are slight. For S / D = 3.0, a vortex street is formed by each tube, and the flow around each tube is independent. The values of $\mathrm{u} / \mathrm{U}$ and $\Delta \mathrm{u} / \mathrm{U}$ for the tubes under the "with rods' condition are lower than those for the 'without rods' condition.

\subsection{Local and overall heat transfer}

The time averaged local $\mathrm{Nu}$ distributions of the first, second, and third tubes in the five-tube arrangements with and without rods for various gap ratios are shown in Figure 8 . The fourth and fifth tubes are omitted, because the first and fifth and second and fourth tubes are symmetric for third tube.

For $\mathrm{S} / \mathrm{D}=1.05$, the distribution of the third tube is symmetric for $\phi=0^{\circ}$, because it is positioned in the center of the row. The distribution has peaks at approximately $\phi= \pm 80^{\circ}$ because of the reattachment of the separated shear layer from the rod. The increase in $\mathrm{Nu}$ is small for the first and second tubes, and the heat transfer of primarily the third tube (center tube) is enhanced.

For $\mathrm{S} / \mathrm{D}=1.5, \mathrm{Nu}$ increases for the entire surface of the second and third tubes and for the rear face of the first tube because the separated shear layer from the rod reattaches on the front face of the tube and the gap flow between the tubes reattaches on the rear face of the tube. The heat transfer enhancement is increased in order of the third, second, and first tubes. 


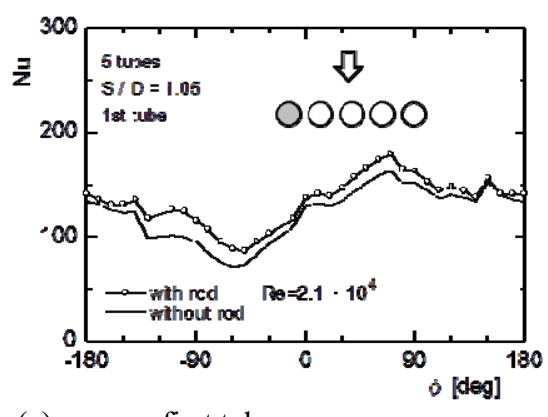

(a)

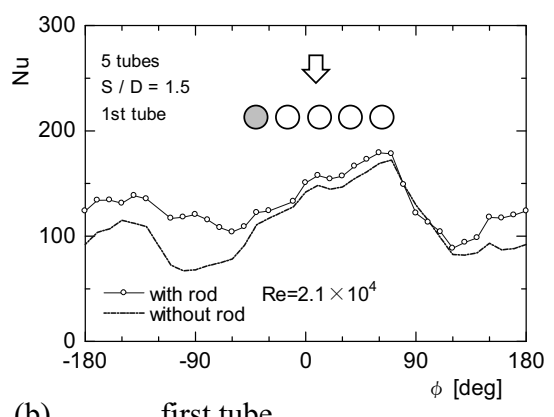

(b)

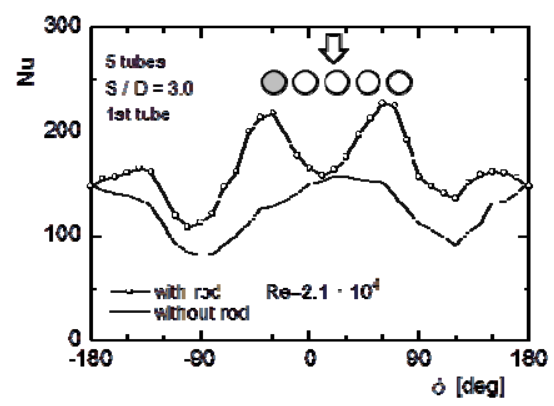

(c)

first tube

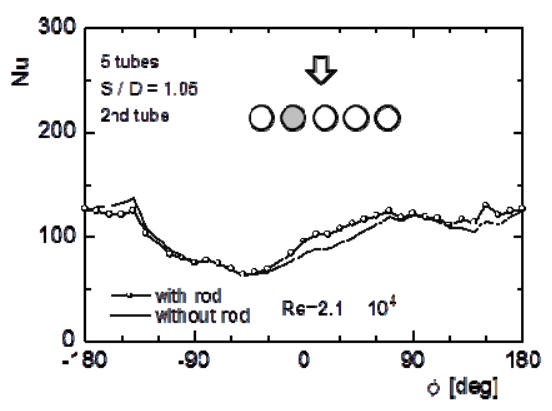

second tube

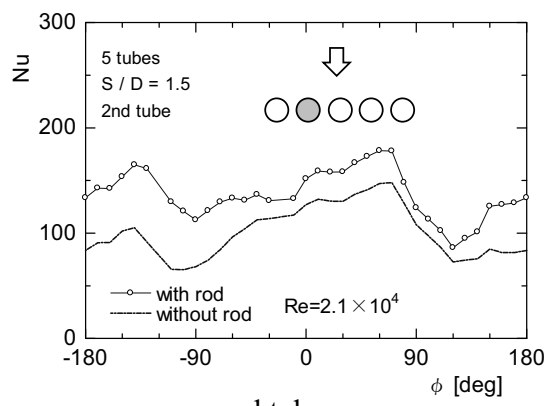

second tube

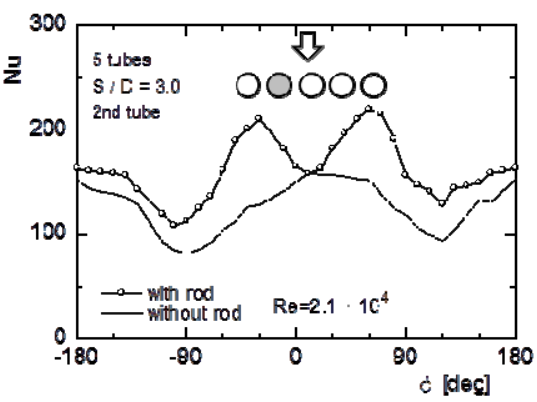

second tube

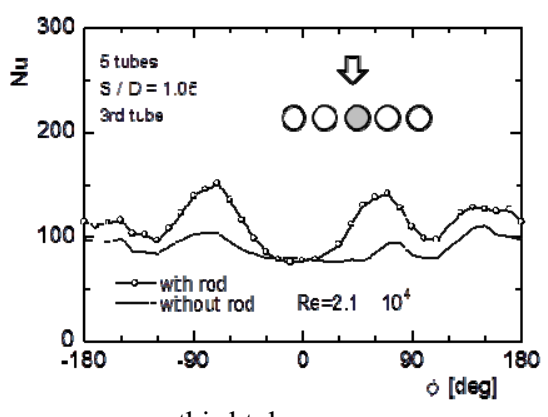

third tube

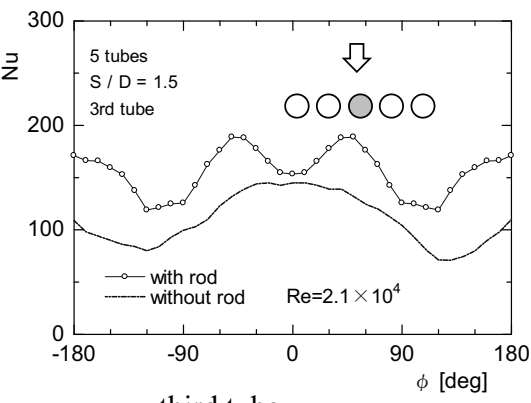

third tube

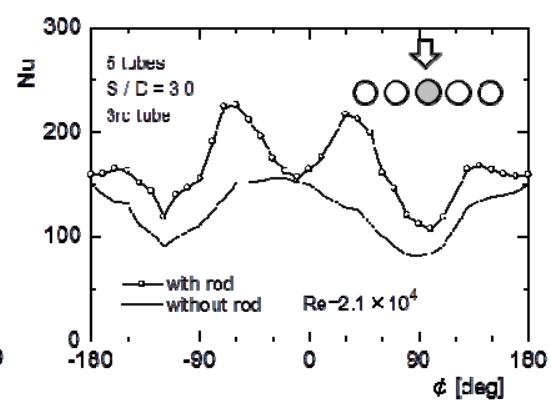

third tube

Fig. 8. Nusselt number distribution (effect of pitch in the five-tube system, $D=20 \mathrm{~mm}, \mathrm{Re}=2.1 \times 10^{4}$ ) for (a) $\mathrm{S} / \mathrm{D}=1.05$, (b) $\mathrm{S} / \mathrm{D}=1.5$, and $(\mathrm{c}) \mathrm{S} / \mathrm{D}=3.0$.

For $\mathrm{S} / \mathrm{D}=3.0$, the distributions of the first, second, and third tubes are the same as that of a single tube. For the 'with rods' condition, all of the tubes in a row have enhanced heat transfer.

\subsection{Pressure distribution}

Figure 9 shows the $\mathrm{Cp}$ distributions under the same conditions as those of Figure 8.

For $\mathrm{S} / \mathrm{D}=1.05, \mathrm{Cp}$ is high in the region of $\phi= \pm$ $90^{\circ}$ for the second and third tubes, because the incoming flow was stagnated by the row. There is little difference between the $\mathrm{Cp}$ distributions of the first, second, and third tubes for the 'with rods' and 'without rods' conditions.

The values of $\mathrm{Cp}$ on the side and rear faces of the tubes for $\mathrm{S} / \mathrm{D}=1.5$ become lower than those for $\mathrm{S} / \mathrm{D}=$ 1.05 , because of the gap flow through the narrow gap between the tubes.
For $\mathrm{S} / \mathrm{D}=3.0$, the $\mathrm{Cp}$ distributions of the first, second, and third tubes are the same as that of a single tube.

\subsection{Heat transfer enhancement and drag reduction}

We also evaluated the effectiveness of applying this technique to multiple tubes arranged in a single row.

The average Nu ratio, NuR / Nuo, of the row of tubes is shown in Figure 10. Subscripts R and O indicate 'with rods' and 'without rods', respectively. The value of $\mathrm{NuR} / \mathrm{Nuo}$ increases as the number of tubes and the size of the gap increase.

Figure 11 shows the drag coefficient ratio, CDR / $\mathrm{CDo}$, of the row of tubes. Based on the drag and momentum balance principle, the drag coefficient was obtained by integrating the velocity distribution of the wake. For the narrow gap ( $\mathrm{S} / \mathrm{D}=1.05)$, the effect on 


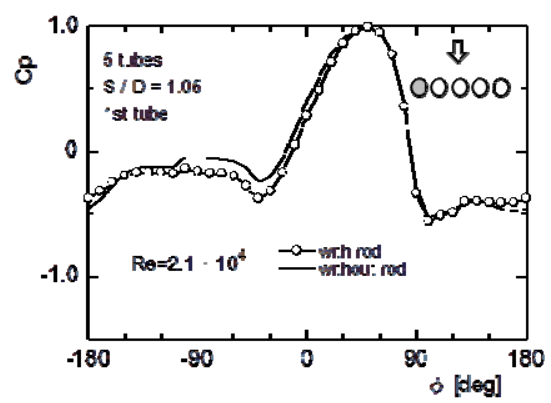

(a)

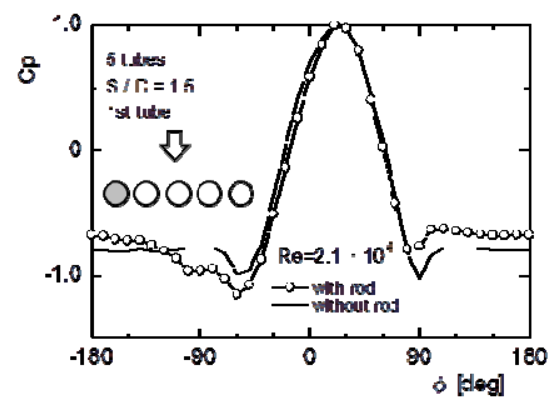

(b)

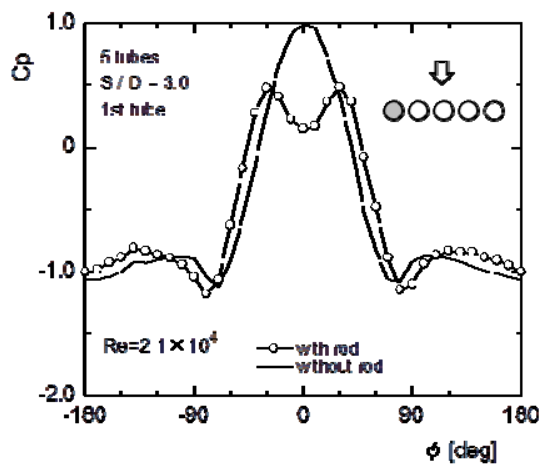

(c)

first tube

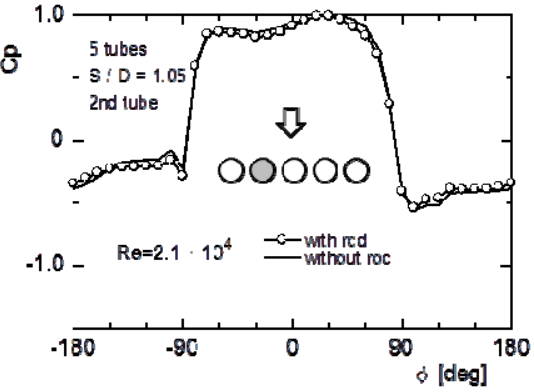

second tube

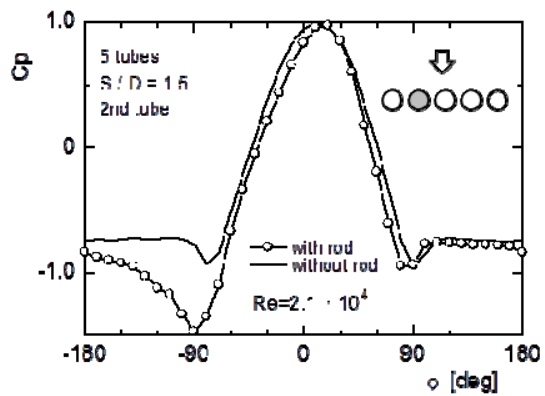

second tube

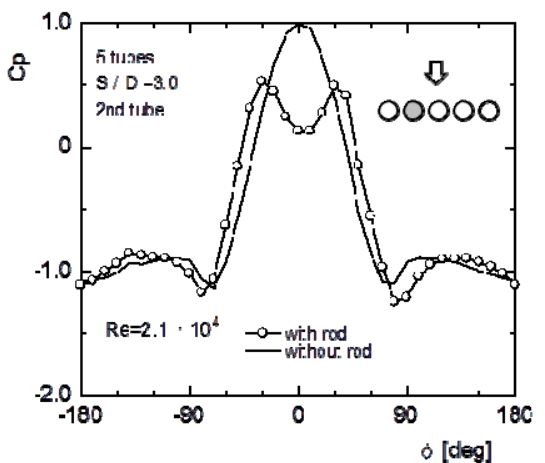

second tube

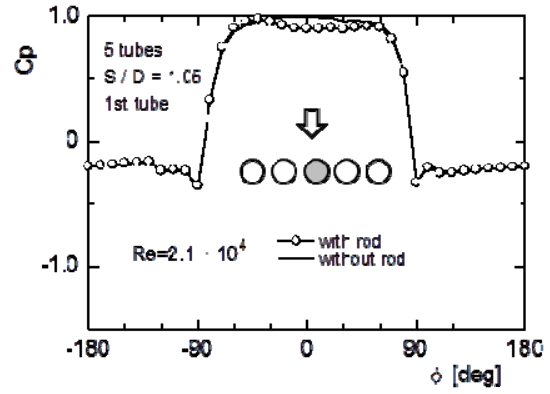

third tube

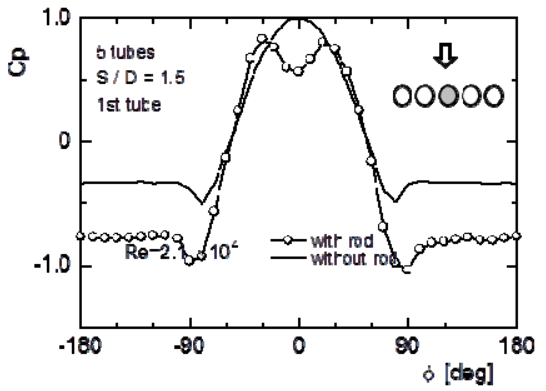

third tube

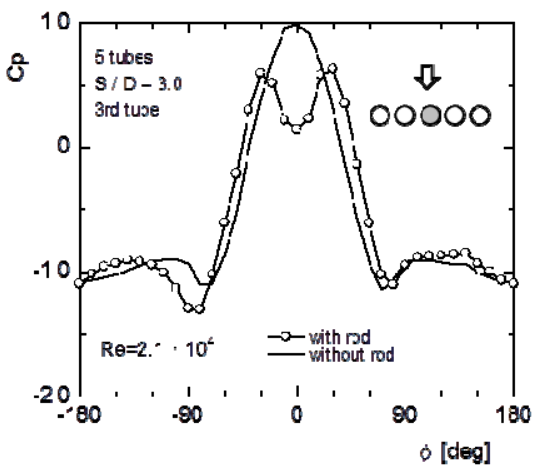

third tube

Fig. 9. Pressure coefficient distribution (effect of pitch in the five-tube system, $D=20 \mathrm{~mm}, \mathrm{Re}=2.1 \times 10^{4}$ ) for (a) $\mathrm{S} / \mathrm{D}=1.05$, (b) $\mathrm{S} / \mathrm{D}=1.5$, and (c) $\mathrm{S} / \mathrm{D}=3.0$.

drag reduction is slight. The value of CDR / CDo decreases as the number of tubes and the size of the gap increase.

The results for the overall evaluation of heat transfer enhancement and drag reduction (NuR / Nuo) / (CDR / CDo) are shown in Figure 12. A higher value of (NuR / Nuo) / (CDR / CDo) indicates higher efficiency. Increasing the number of tubes and the size of the gap results in higher efficiency.

\section{Conclusions}

A row of small rods was placed upstream from a row of circular tubes in order to reduce drag and enhance heat transfer.

Experiments were performed to confirm the effectiveness of this technique. The experimental results revealed the effectiveness of this technique for enhancing the local heat transfer of a row of circular tubes. Moreover, increasing the number of tubes and the gap between the rows of rods and tubes provided greater heat transfer enhancement and increased drag reduction. 


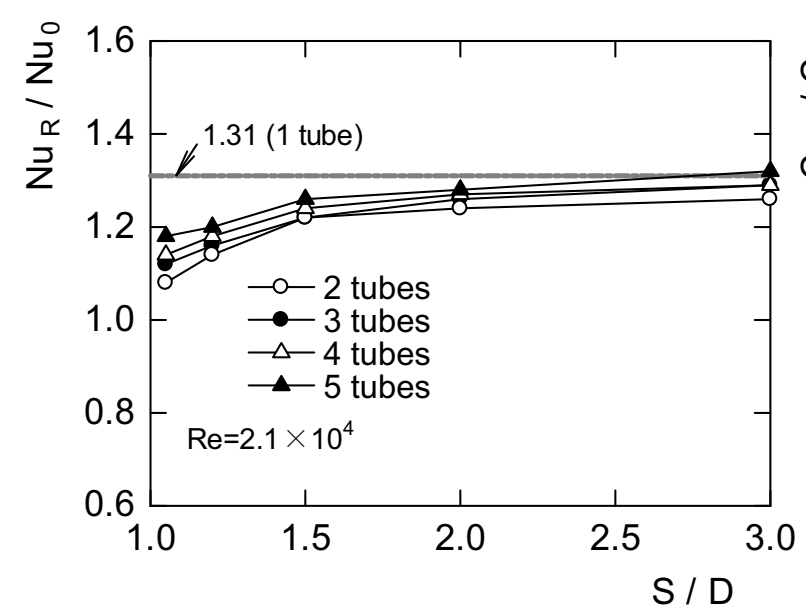

Fig. 10. Average Nusselt number of the row of tubes

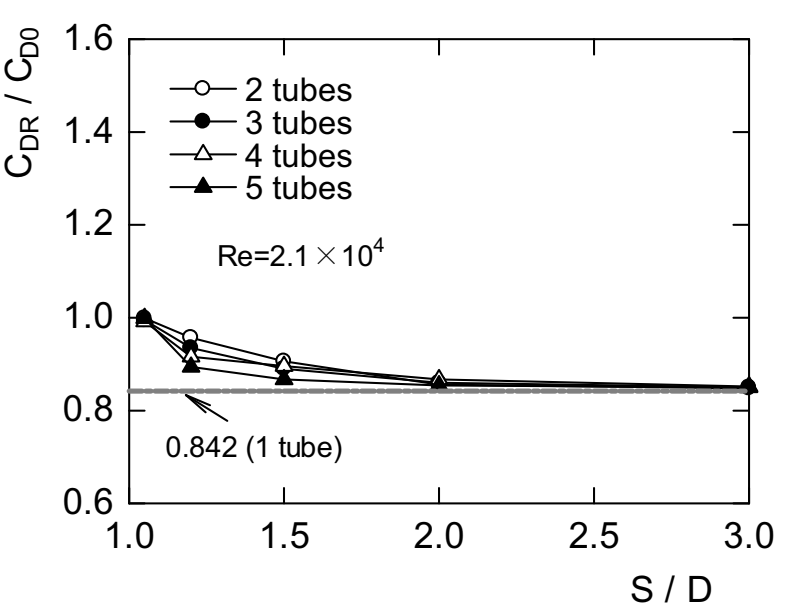

Fig. 11. Drag coefficient of the row of tubes

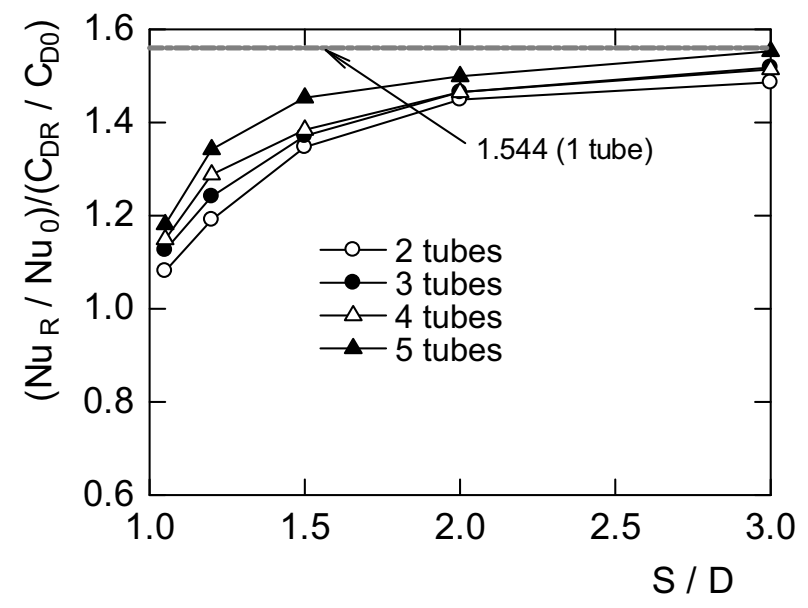

Fig. 12. Heat transfer enhancement and drag reduction

\section{References}

1. Wen-Chieh Huang, Cheng-An Chen, Chi Shen, Jung-Yang San, Effects of characteristic parameters on heat transfer enhancement of repeated ring-type ribs in circular tubes, Exp. Thermal and Fluid Science. 68, pp. 371-380 (2015)

2. Subhankar Saha, Sujoy Kumar Saha, Enhancement of heat transferof laminar flow through a circular tube having integral helical rib roughness and fitted with wavy strip inserts, Exp. Thermal and Fluid Science. 50, pp. 107-113 (2013)

3. K. M. Kwak, K. Torii, K. Nishino, Simultaneous heat transfer enhancement and pressure loss $\mathrm{T}$ reduction for finned-tube bundles with the fiest or two transverse rows of built-in winglets, Exp. Thermal and Fluid Science. 29, pp. 625-632 (2005)
4. T. Tsutsui, T. Igarashi, Heat Transfer Enhancement of a Circular cylinder, Trans. ASME J. Heat Transfer. 128, pp. 226-233 (2010)

5. T. Tsutsui, T. Igarashi, Drag Reduction of a Circular cylinder in an Air-stream, J. Wind Engineering and Industrial Aerodynamics, 90, pp. 527-541 (2002)

6. M. M. Zdravkovich, Flow Around Circular Cylinders Vol.2: Applications (Oxford University Press, 2005)

7. Tsutsui, An Experimental Study on Heat Transfer around Two Side-by-side Closely Arranged Circular cylinders, Trans. ASME J. Heat Transfer. 132, pp. 111704-1-111704-8 (2010) 